\title{
Experimental investigation of adhesive-based self-healing of cementitious materials
}

\author{
C. Joseph*, A. D. Jefferson*, B. Isaacs*, R. Lark* and D. Gardner* \\ Cardiff University
}

This paper presents the results of a series of self-healing experiments conducted on reinforced mortar beams containing adhesive-filled glass reservoirs. An overview of the findings of the preliminary investigation stage of experiments is given in addition to the results of a parametric study which investigates the effect of the level of reinforcement and loading rate on the amount of self-healing. Results show that both primary and secondary healing occurs during the first and second loading cycles respectively. Qualitative results also show clear evidence of the occurrence of crack-healing following the first loading cycle and new crack formation during the second loading cycle. The long-term motivation for this work is to provide data suitable for the development of a numerical material model for the autonomic healing process in cementitious materials.

\section{Introduction}

Cementitious materials such as concrete are the most widely used man-made materials on the planet, and cement is used to make approximately 2.5 metric tonnes of concrete per person alive per year (van Oss, 2005). In addition, almost half of the $£ 80$ billion spent on construction work in the UK per annum, of which concrete forms a large portion, is allocated to repair and maintenance of existing structures (DTI, 2006). The durability of concrete is therefore a very important issue, which requires addressing. Cracking caused by shrinkage effects during curing and mechanical loading during the subsequent service life of the structure accelerate many of the degradation processes affecting concrete durability, such as reinforcement corrosion and freeze-thaw action.

Traditionally the durability of concrete structures has been addressed by the initial material specification and reinforcement or prestressing detailing. Deterioration of the structure is then addressed in a reactive manner by way of maintenance regimes. Recently material scientists have begun to question the notion of a fixed unchanging design specification and, often gaining inspiration from biology, have begun to develop materi-

* Research Office, Cardiff School of Engineering, Queen's Buildings, The Parade, Newport Road, Cardiff CF24 3AA, UK

(MACR 900096) Paper received 12 June 2009; last revised 15 December 2009; accepted 12 March 2010 als which are capable of adapting to their environment (van der Zwaag, 2007). Self-healing materials, which have the ability to detect and respond to damage through various processes, thereby recovering certain material properties, are one such area of material development.

Self-healing materials, which are a category of smart material, can be divided into two areas: autogenic and autonomic healing materials. Traditional concrete, in which ongoing hydration is found to close early-age microcracks, exhibits a 'natural' healing phenomenon termed autogenic healing, since it is generic to the material. Techniques have been developed to enhance such autogenous healing, which involve adding soluble capsules of cement and/or water to a concrete mix to enhance autogenous healing after early-age curing, for example (Kepler et al., 2001). When the healing behaviour is specifically engineered to occur within a composite material system, such as that discussed within this paper, the resulting 'manufactured' healing response is termed autonomic healing (Joseph, 2008; White et al., 2001).

The autonomic healing concept investigated in this paper is shown in Figure 1. This was originally proposed for cementitious materials by Dry $(1994,2006)$ and is similar to that investigated in polymeric materials by White et al. (2001).

The concept is based on the principle that cracks which form in the cementitious matrix also cause brittle adhesive-filled capsules or tubes, embedded within the matrix, to crack. The adhesive is thereby intended to be 
(a)

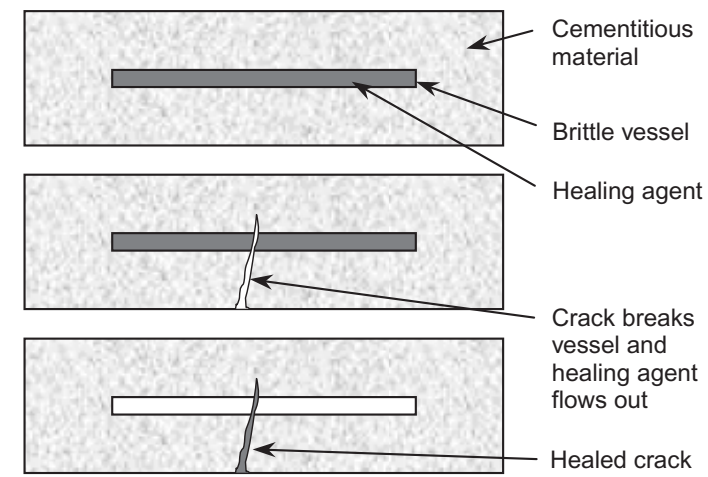

Figure 1. Autonomic healing concept in cementitious materials

released into the crack plane, cure, and thus heal the original crack through bonding of the crack faces. This concept has also been explored by other authors including Li et al. (1998), Mihashi et al. (2000) and more recently by Nishiwaki et al. (2006).

It is worth mentioning that techniques which repair and seal cracks with resins are widely used in practice; however, these require intervention once cracks have formed and thus are conceptually different from the self-healing systems considered in this paper.

The long-term motivation for the work undertaken at the Cardiff School of Engineering is to establish a numerical material model for the autonomic healing process in cementitious materials. Since experimental data on this relatively new self-healing material are limited, a parameter investigation was deemed necessary for which comprehensive data would be available. These data included

(a) width, nature and location of cracks

(b) viscosity and degree of migration of adhesive

(c) strength and stiffness of specimen pre- and posthealing.

The efficacy of self-healing within a cementitious material may be measured in terms of various parameters including permeability, acoustic emissions and mechanical strength. This paper examines the autonomic-healing ability of reinforced mortar beams containing adhesive-filled glass reservoirs. Healing ability is examined in the context of mechanical strength recovery, or gain, for specimens subjected to three-point bending. Particular focus has been given to the effect of reinforcement level and the rate of loading on the efficacy of the self-healing system.

\section{Preliminary investigations}

A substantial amount of preliminary experimental work has been completed prior to the development of a successful self-healing experimental method, as outlined by Joseph et al. (2007) and discussed in detail by
Joseph (2008). Various aspects of the experimental method have been investigated including

(a) the type of healing agent

(b) the delivery system

(c) the mortar mix design

(d) the quantity of steel reinforcement used.

\section{Healing agent}

The three main autonomic healing agents which have been investigated in the literature to date are

(a) epoxy resins (Mihashi et al., 2000)

(b) cyanacrylates (Li et al., 1998)

(c) alkali-silica solutions (Mihashi et al., 2000).

In addition to being readily available and cost-effective, a suitable agent should have the ability to be readily encapsulated within an internal or external supply system and be sufficiently mobile to allow migration to the areas of damage following release. In addition, crack reopening should be resisted post-healing, and therefore the agent should have sufficient mechanical properties after curing, ideally equal to or greater than the properties of the cementitious matrix. In order to improve long-term durability the agent should also have sufficient longevity and compatibility with the cementitious matrix, over the lifetime of the structure; however, research has not focused on this area to date.

Several healing agents including epoxy resins and cyanoacrylates have been examined during the feasibility stage of the experimental development. These have been assessed based upon their viscosity, curing period, their mechanical ability to bond both smooth and rough concrete surfaces together, and their longevity within an encapsulated system. Epoxy resins are two-part systems which cure in the absence of oxygen and are therefore unsuitable for internal encapsulation or for use in an external circulatory supply system, where good post-encapsulation longevity (i.e. extended potlife) is required. Mihashi et al. (2000) examined the feasibility of separate storage of the two epoxy resin components, adjacent to one another within a cementitious matrix, although the authors concluded that insufficient mixing on release resulted in poor curing and therefore poor mechanical performance of the adhesive. Cyanoacrylates (superglue) are more suitable healing agents since they are single-agent adhesives with generally lower viscosities than epoxy resins, and are therefore able to flow into, and heal, finer cracks.

Rite-Lok EC-5 (3M, 2007) cyanoacrylate was selected for use in the final parametric study because of its suitability at bonding a wide range of substrates including ceramics, and for its ability to infiltrate microcracks owing to capillary effect as a result of its extremely low viscosity (typically 5 centipose). The adhesive was also found to gain strength rapidly during curing, and achieve a final tensile strength which was significantly greater than the parent cementitious ma- 
trix. Rite-Lok EC-5 has a full cure time of $24 \mathrm{~h}$ and a tensile strength (ISO 6922) of $20 \mathrm{MPa}$ after curing. It should be noted that Rite-Lok EC-5 is an ethyl cyanoacrylate monomer which is stabilised through the addition of a weak acid. When the adhesive contacts a surface, trace amounts of water (specifically hydroxide ions) neutralise the acidic stabiliser in the adhesive, resulting in rapid anionic polymerisation of the cyanoacrylate.

\section{Delivery system}

The two main forms of delivery system are

(a) internal encapsulation of the adhesive in brittle capsules (Li et al., 1998)

(b) external delivery of the adhesive through a brittle supply network (Mihashi et al., 2000).

Preliminary investigations have focused on the use of internal encapsulation using hollow capillary tubes, as illustrated in Figure 2.

Tubes $75 \mathrm{~mm}$ long with $0.8 \mathrm{~mm}$ ID (internal diameter) and $1.5 \mathrm{~mm} \mathrm{ID}$, and $100 \mathrm{~mm}$ long tubes of $3 \mathrm{~mm}$ ID were internally encapsulated within prismatic mortar beams during the preliminary experiments. The details for these tubes are given in Table 1 .

Two different configurations of capillary tubes were explored: (a) a single layer of 5 No. tubes and $(b)$ a double layer of ten tubes (two layers of 5No.), placed centrally in the beam, as illustrated in Figure 3.

In order to minimise the amount of air trapped with-

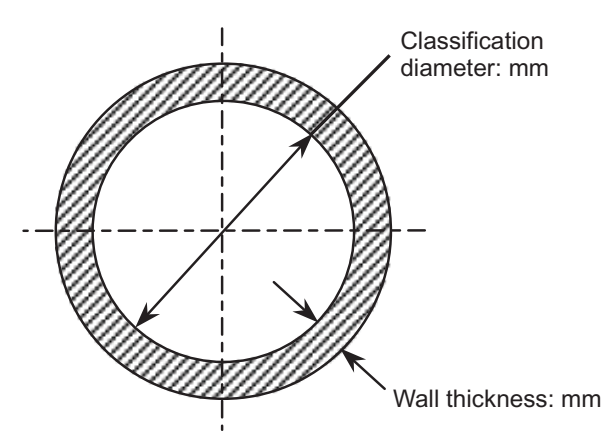

(a)


(b)

Figure 2. (a) Borosilicate capillary tube cross-sectional details and (b) pictures of $1.5 \mathrm{~mm}$ and $3.0 \mathrm{~mm}$ diameter tubes filled with cyanoacrylate and plugged with wax in the tubes they were filled with adhesive by rotating the tube to a near horizontal position and using the capillary attractive force of the tube itself to draw up the liquid. The ends were then sealed by inserting the tube into a soft wax compound. In the control specimens, the adhesive was replaced with ink.

In order to ensure sufficient compaction of the mortar around the capillary tubes, tubes were placed manually in layers, although this did result in some variation in the final placement, as shown in Figure 4. In order to minimise tube breakages, and provide a sufficient supply of agent to the crack face, and also reduce the capillary resistive force of the tube walls on the adhesive as it flowed out into the crack, the largest $3 \mathrm{~mm}$ ID tubes were used in the final experimental series.

\section{Mortar mix design}

Small-scale laboratory beams $(75 \times 75 \times 255 \mathrm{~mm})$ were used during the entire experimental programme (Figure 3). In order to achieve a more homogeneous material at this specimen scale the beams were cast using mortar rather than concrete. The maximum aggregate size was limited to $2 \mathrm{~mm}$, and the maximum aggregate to specimen dimension ratio was therefore 1:35. A series of trial mixes were undertaken, and a ratio of $0 \cdot 6: 1: 3 \cdot 5$ (water:ordinary Portland cement (OPC):sand), by weight, was found to offer suitable workability and allow sufficient material compaction around the reinforcing bar and capillary tubes. This mix design was used for all subsequent sets of experiments (apart from set 1), as shown in Table 3, see later.

\section{Steel reinforcement}

All mortar beams were reinforced in order to control the rate of crack opening in the specimens during three-point bend tests, and to examine the efficacy of the self-healing system within a reinforced cementitious material. The nominal minimum level of reinforcement specified in Eurocode 2 (CEN, 2004) for reinforced concrete beams is typically $0 \cdot 14 \% b d$, where $b$ is the beam breadth and $d$ is the depth to the centre of the tensile reinforcement. In order to meet this requirement, a smooth $3.15 \mathrm{~mm}$ diameter high-yield steel reinforcing bar was used in the preliminary experiments. Higher levels of reinforcement were also examined during both the preliminary and final experimental programme, as described in the section 'Experimental work'. The maximum percentage of reinforcement used was $0.7 \%$, which was provided by a $6.7 \mathrm{~mm}$ diameter high yield steel bar.

Three $250 \mathrm{~mm}$ long samples of the $3.15 \mathrm{~mm}$ diameter and the $6.7 \mathrm{~mm}$ diameter steel bar were tested in a tensile testing machine in accordance with the recommendations of BS 4449 (BSI, 2005). The mean mechanical properties for both bar materials, together with the coefficients of variation $(\mathrm{CoV})$, are given in Table 2 . 
Table 1. Specifications of capillary tubes used in preliminary experiments

\begin{tabular}{|c|c|c|c|}
\hline Classification diameter: $\mathrm{mm}$ & $0 \cdot 8$ & $1 \cdot 5$ & $3 \cdot 0$ \\
\hline Wall material & \multicolumn{3}{|c|}{ Borosilicate glass } \\
\hline Wall thickness: mm & - & - & $0 \cdot 5$ \\
\hline Length: mm & 75 & 75 & 100 \\
\hline Total internal capacity of single tube, excluding wax end plugs: $\mu$ l & 34 & 118 & 650 \\
\hline
\end{tabular}

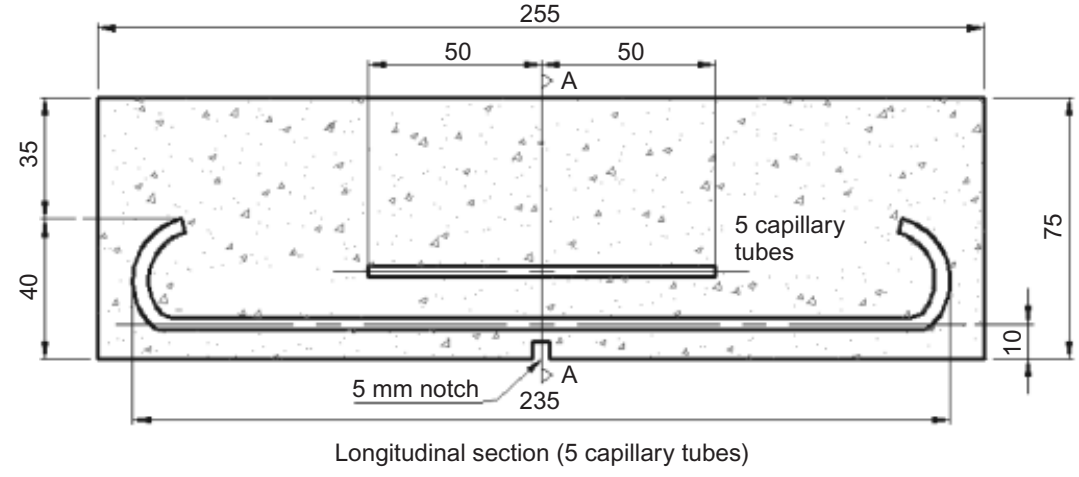

(a)

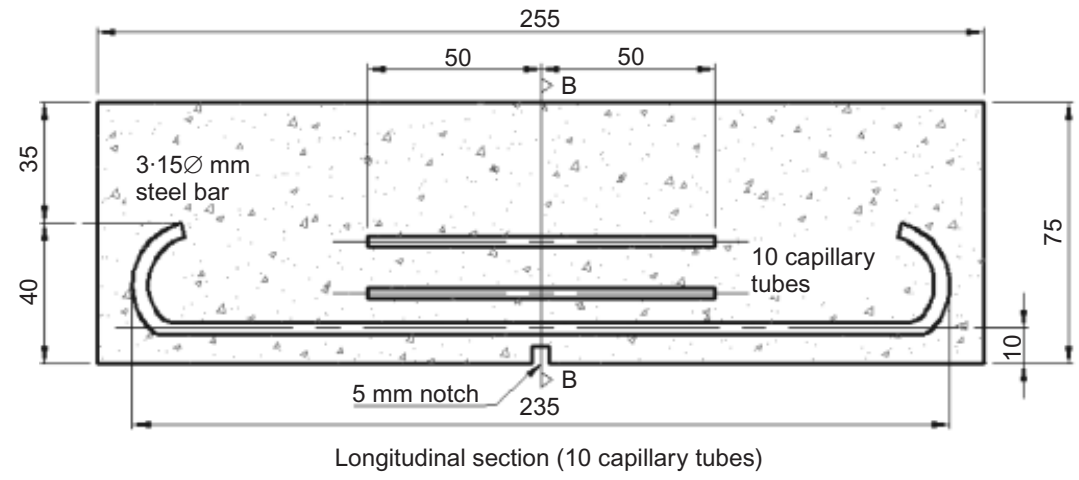

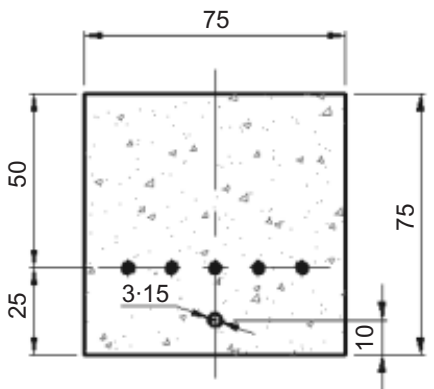

Section A-A (5 capillary tubes)

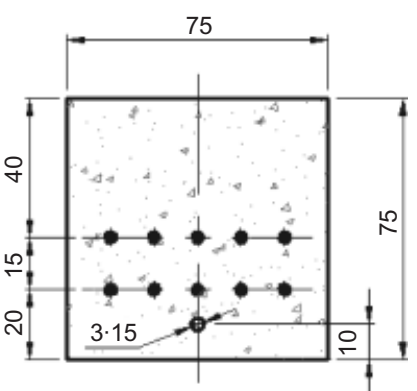

Section B-B (10 capillary tubes)

(b)

Figure 3. Specimen configuration for the two sets of preliminary experiments containing (a) a single layer and (b) a double layer of $5 \mathrm{No} .100 \mathrm{~mm}$ long, $3 \mathrm{~mm}$ diameter glass capillary tubes

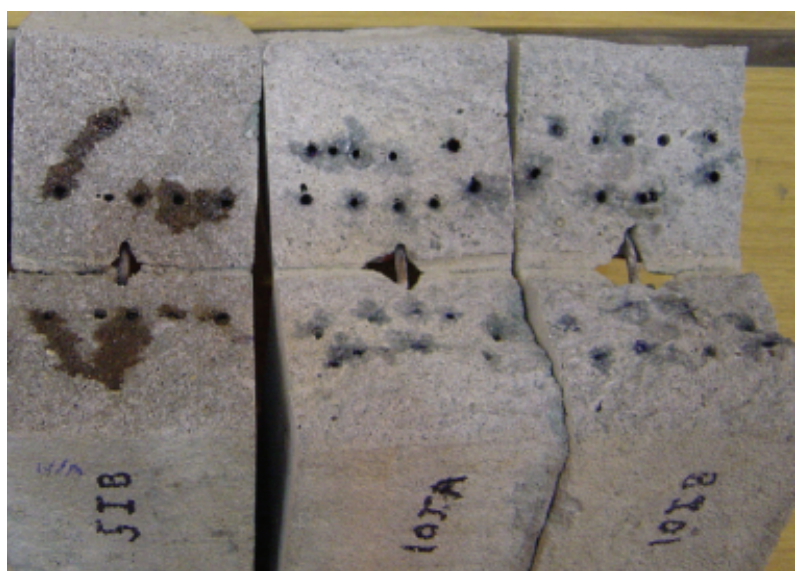

Figure 4. Ink staining on crack faces of control specimens from preliminary experiments
Summary of preliminary investigation work

The preliminary investigation work as outlined above was concluded with the completion of two sets of experiments. These consisted of two sets of six beams, with each set comprising four self-healing ( $\mathrm{SH}$ ) and two control (C) beams, tested under three-point loading. The capillary tube and reinforcement configuration for both sets of experiments is shown in Figure 3.

All beams were subjected to a two-stage, three-point bending, test procedure, in an identical manner to that undertaken in the final experimental procedure described in the section on testing procedure. The tubes in the SH beams were filled with Rite-Lok EC5 cyanoacrylate, and the tubes in the control beams were filled with ink, before being sealed with wax plugs, prior to casting.

Magazine of Concrete Research, 2010, 62, No. 11 
Table 2. Summary of the mean mechanical properties for the $3.15 \mathrm{~mm} \phi$ and the $6.7 \mathrm{~mm} \phi$ high yield steel reinforcement bars (CoV given in brackets)

\begin{tabular}{l|l|c|c|c|c}
\hline Diameter: mm & Profile & Elastic modulus: GPa & $0 \cdot 2 \%$ proof stress: MPa & Ultimate strength: MPa & Elongation at failure: \% \\
\hline $3 \cdot 15$ & Smooth & $205 \cdot 2(0 \cdot 9 \%)$ & $563 \cdot 1(0 \cdot 7 \%)$ & $597 \cdot 3(2 \cdot 1 \%)$ \\
$6 \cdot 70$ & Smooth & $192 \cdot 3(1 \cdot 7 \%)$ & $526 \cdot 1(1 \cdot 2 \%)$ & $576 \cdot 3(0 \cdot 6 \%)$ \\
\hline
\end{tabular}

The main conclusion from these tests was that, despite some evidence of a small amount of healing in one of the four SH beams containing a double layer of tubes, the glue had not been drawn into the cracks in sufficient quantity to achieve healing. This was confirmed by the very limited extent of ink penetration which was observed on the crack faces of the control beams, as illustrated in Figure 4. The extent of glue flow observed on the crack faces of the SH beams was even less owing to its higher viscosity, and large quantities of both liquid glue and ink were found to remain in both sections of the capillary tubes following complete fracture of all specimens.

The negative pressure forces created by the wax plugs at either end of the capillary tubes are believed to be responsible for the inadequate release of glue and ink into the crack. The capillary attractive force of the crack and the gravitational force on the fluid mass are insufficient to overcome the capillary resistive force of the glass tube, and the negative pressure force caused by the sealed ends (Figure 5). The small degree of ink flow on the crack faces, as shown in Figure 4, is therefore probably attributable to ink released from the local vicinity of the crack site as the glass tube fractures. Since the crack propagates in a direction perpendicular to the orientation of the tube, the amount of adhesive released locally is minimal compared with the volume remaining in the tube. It should be noted that this is different from the case of healing within engineered cementitious composites (ECCs) (Li et al., 1998) where tubes have multiple cracks, and the case of self-healing within laminated composites (Williams et al., 2007), where the tubes are sheared along their lengths as a result of the delaminating mode of failure.

To overcome the above difficulties, longer capillary tubes were used in the final experimental set-up (see

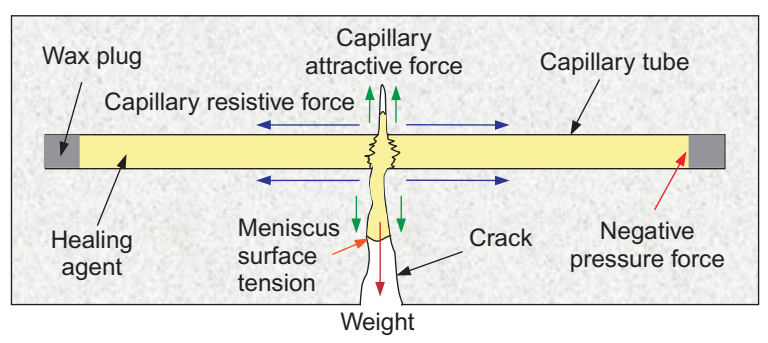

Figure 5. Schematic illustration of the main forces acting on an internally encapsulated healing agent section on experimental procedure) which extend outside of the mortar beam and are open to the atmosphere, thus removing the resistance provided by the end plugs.

\section{Experimental work}

\section{Programme of study}

The experimental programme of study reported in this paper comprised five sets of experiments as outlined in Table 3. The effect of the level of reinforcement and the rate of machine stroke monotonic loading on the efficacy of the self-healing behaviour was examined. Owing to unforeseen mechanical failure of the testing equipment, sets 4 and 5 were tested at 70 days rather than the 28 days chosen for sets $1-3$. This did nevertheless allow the effect of the specimen age on the autonomic-healing ability of the beams to be examined.

\section{Experimental procedure}

The self-healing and control specimens for each of the experimental sets (Table 3) were prepared in a similar manner to the first set of tests on lightly reinforced notched beams, as illustrated in Figure 6 .

The set 1 beams were reinforced with a single $3.15 \mathrm{~mm}$ diameter high yield steel bar placed on $10 \mathrm{~mm}$ brick spacing blocks, as shown in Figure 7 . Since the bar had a smooth profile, anchorage was provided by bending the bar at either end (Figure 6). Experimental sets 2 and 5 had identical reinforcement arrangements, but for set 4 the $3.15 \mathrm{~mm}$ bar was replaced with a larger $6.7 \mathrm{~mm}$ diameter bar, and for set 3 the single $3.15 \mathrm{~mm}$ bar was replaced with two $3.15 \mathrm{~mm}$ bars, spaced at a horizontal distance of $30 \mathrm{~mm}$ apart.

The healing agent, for all $\mathrm{SH}$ specimens in all experimental sets, was supplied by way of four No. $3 \mathrm{~mm}$ diameter hollow capillary tubes, placed in a single layer, $20 \mathrm{~mm}$ from the bottom of the beam (Figures 6 and 7). These tubes were inserted through lubricated pre-drilled holes in the mould end plates prior to casting, as illustrated in Figure 7. The capillary tubes were open to the atmosphere on one side and plugged with wax on the other side for the first set of tests on notched, lightly reinforced beams (Figure 6). The capillary tubes for all beams in remaining sets, however, had open ended supply tubes fixed to both ends of the 


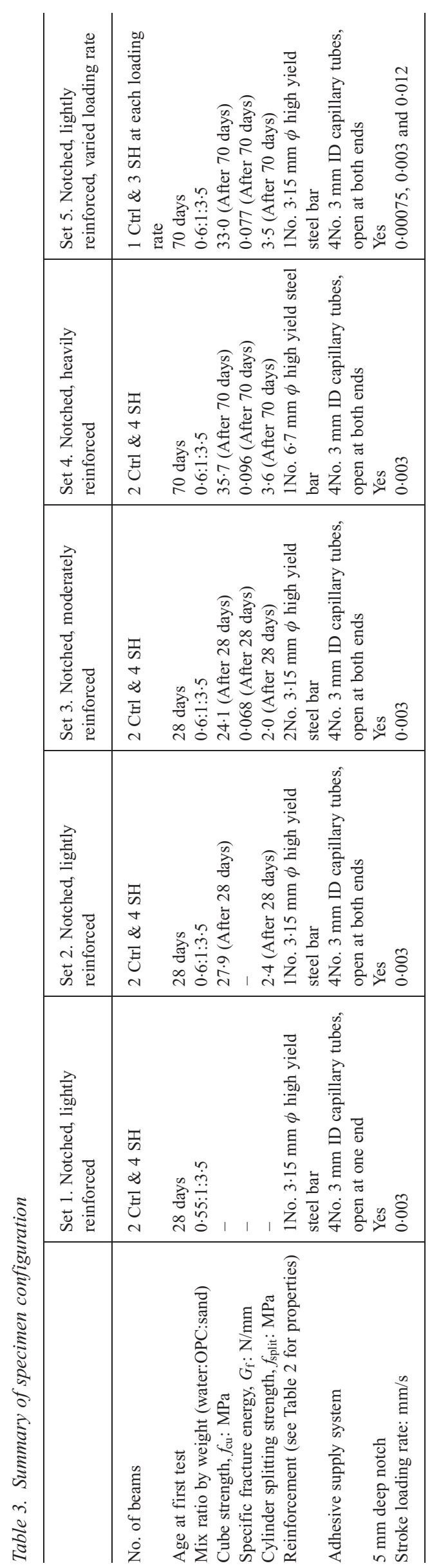

specimen, in order to maximise the provision of healing agent to both crack faces during testing.

All specimens were demoulded $24 \mathrm{~h}$ after casting, and then cured for 28 or 70 days in air, prior to being tested. A $5 \mathrm{~mm}$ deep notch was sawn on the underneath of all beams at the mid-point prior to testing (Figure 6).

\section{Testing procedure}

All of the capillary tubes within the SH specimens were filled with Rite-Lok EC5 cyanoacrylate to a level of $25 \mathrm{~mm}$ above the centreline of the tube prior to testing (Figure 6). In order to minimise air voids, adhesive was injected into the tubes using a syringe. The four capillary tubes in each of the two control beams were also filled and in an identical manner, but with a low viscosity ink (approx. 3 centipose) rather than adhesive. The amount of adhesive or ink placed in the supply system prior to testing was approximately $10 \mathrm{ml}$ for each specimen, and this was not replenished during testing.

Two cycles of three-point bend testing were completed on each beam under machine stroke control, as shown in Figure 8. The monotonic loading rate for sets 1 to 4 was $0.003 \mathrm{~mm} / \mathrm{s}$, and for set 5 three different loading rates were used - $0.00075 \mathrm{~mm} / \mathrm{s}, 0.003 \mathrm{~mm} / \mathrm{s}$ and $0.012 \mathrm{~mm} / \mathrm{s}$ - in order to examine its effect on self-healing. Crack mouth opening displacement (CMOD) and central deflection were recorded. The latter was measured using a transducer supported by way of an aluminium armature, connected to the centreline of one side of the beam (Figure 8), in order to eliminate 'bedding in' effects.

During the first loading cycle the beam was loaded until it had regained its initial peak strength prior to the glass tubes breaking, and a CMOD value of at least $0.3 \mathrm{~mm}$ had been reached, before being unloaded. A typical response from the first cycle of loading for a $\mathrm{SH}$ and control beam is given in Figure 10, see later.

In the second loading cycle the beams were tested at the same loading rate to failure, or until a central deflection value in excess of $3 \mathrm{~mm}$ was recorded. For the control specimens the second loading cycle was completed immediately after the first loading cycle, but for the SH specimens a delay of $24 \mathrm{~h}$ was imposed to allow full curing of the cyanoacrylate adhesive to occur. A typical response from the second loading cycle for an $\mathrm{SH}$ and control beam is given in Figure 10, see later.

\section{Results and discussion}

The complete three-point bending results for all sets of experiments are given in Joseph (2008). While there is naturally some variability in the experimental results, due to the nature of the self-healing system investigated, several trends and features have been identified, as detailed in following sections. 

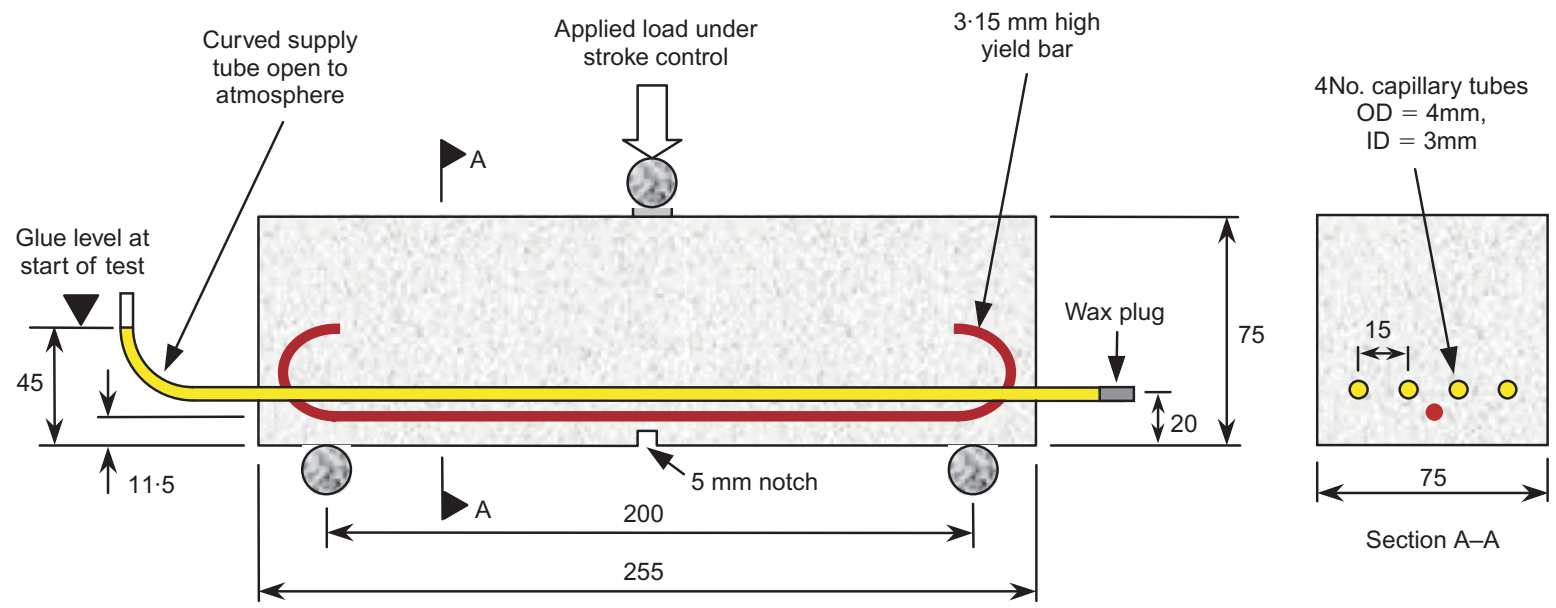

Figure 6. Specimen arrangement for set 1 experiments on lightly reinforced notched beams. (Note: Experimental sets 2-5 have an open curved supply tube at both ends of the specimen)

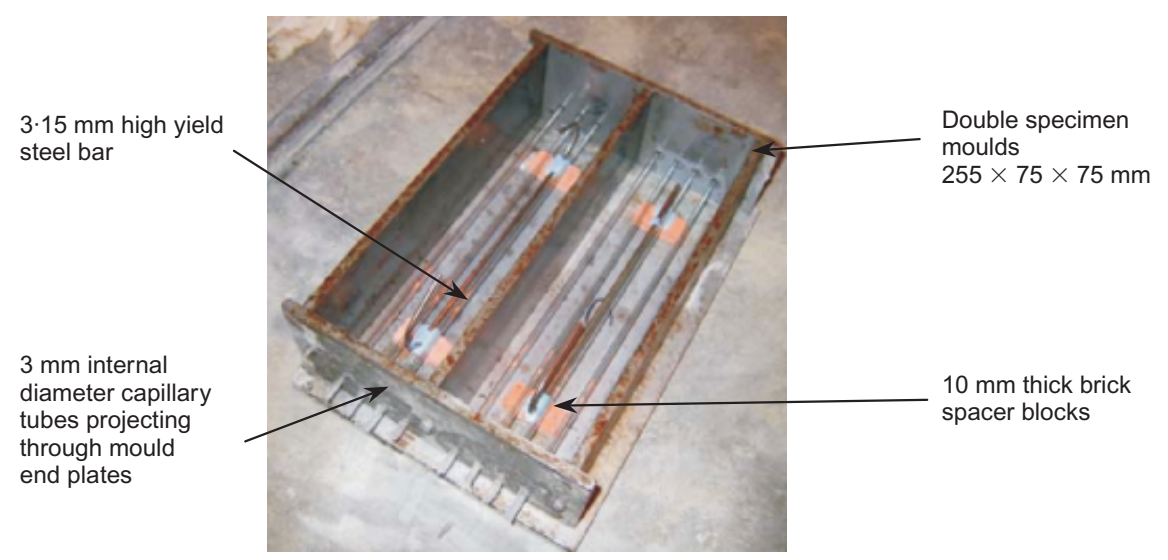

Figure 7. Illustration of glass tube and reinforcement configuration for set 1 beams

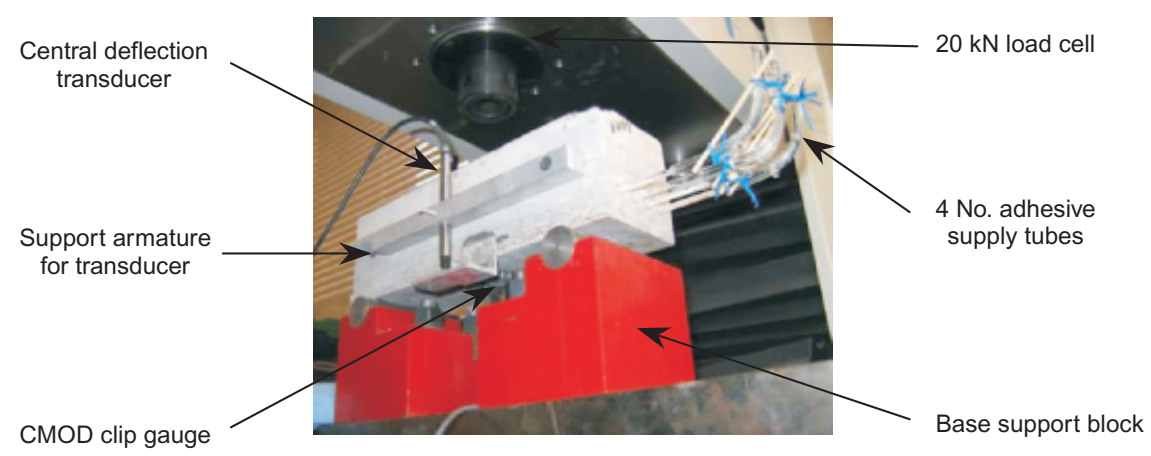

Figure 8. Testing arrangement for set 1 experiments on lightly reinforced notched beams

\section{Typical healing response}

The load-CMOD and load-central deflection response of a representative SH beam and a control beam are presented in Figure 9. It may be seen that, for both the control specimen and the first test on $\mathrm{SH}$ beam 4, there is some pre-peak non-linearity owing to microcracking between about 4 and $5 \cdot 2 \mathrm{kN}$. This is followed by a sudden drop of approximately $0.7 \mathrm{kN}$ over a 


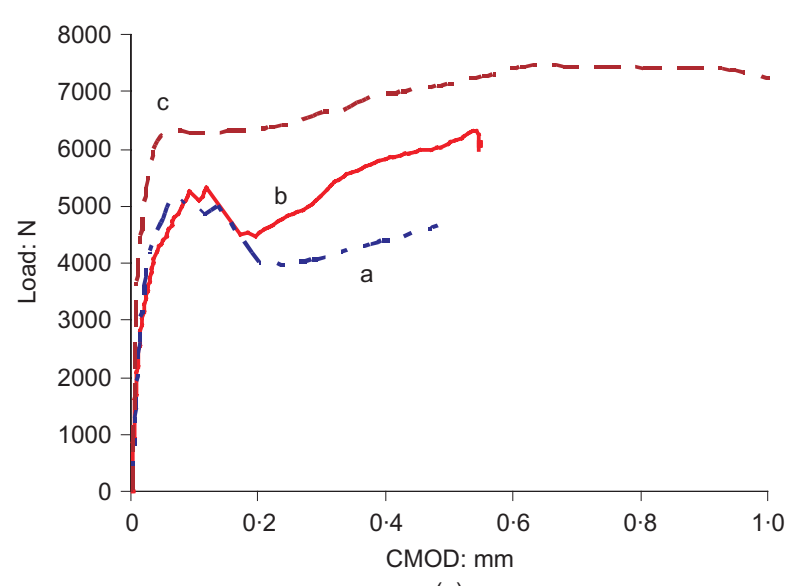

(a)

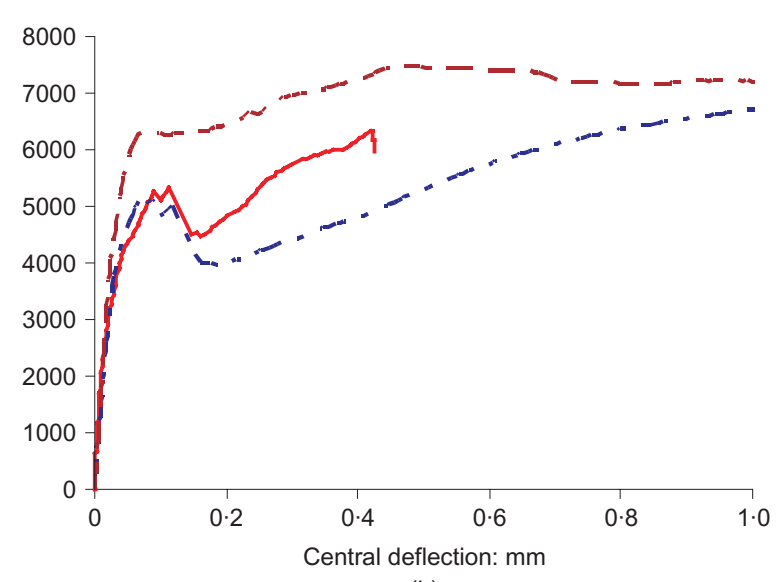

(b)

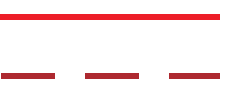

SH beam

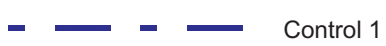

SH beam (healed)

Figure 9. (a) Load-CMOD and (b) load-central deflection response for SH beam 4 and control beam 1 from set 1

CMOD increase of approximately $0 \cdot 1 \mathrm{~mm}$ for the selfhealing beam, compared with about $1 \cdot 1 \mathrm{kN}$ over $0.13 \mathrm{~mm}$ for the control beam. This drop coincides with the brittle fracture of the four borosilicate capillary tubes which emit a distinctive breaking sound during testing. Thereafter, the primary load-carrying mechanism is performed by the steel reinforcement and the stiffness of the control beam is given by the gradient of the line at point ' $a$ ' in Figure 9(a). It should be noted that a lightly reinforced beam without the glass tubes would also show a drop, but it is likely to be less pronounced.

Figure 9 shows that even during the first test on $\mathrm{SH}$ beam 4 there is some evidence of 'primary' healing. This is apparent in the increased gradient at point ' $b$ ' when compared with the equivalent gradient of the control at point ' $a$ '. Cyanoacrylates are acidic solutions which are able to cure rapidly within a period of seconds. The conditions within the mortar, including the presence of moisture and the alkaline environment, further accelerate the curing process, and are believed to be responsible for the rapid primary healing observed in the vast majority of the first cycle tests.

The efficacy of the autonomic healing process may also be evaluated by comparing the second cycle response for the SH beam (healed) with the first cycle response of the SH beam or the control beam. Figure 9 indicates that in addition to the 'primary' healing observed during the first loading cycle there is also clear evidence of a 'secondary' healing effect in the second loading cycle; the gradient of the response up to point ' $c$ ' is stiffer than that of the original control beam, the peak load has increased by over $20 \%$, and the post-peak response is more ductile.

The first cycle unloading response has been recorded for experimental sets 2 to 5 , and therefore permanent set values (CMOD values on unloading) are known. This allows the efficacy of the self-healing system to be examined in a slightly different manner; that is, by comparing the mechanical response of the 'healed' beam to that of the control beam during the second loading cycle.

The difference between the two methods of examining the efficacy of the self-healing process may therefore be attributed to whether or not the second loading cycle results for the SH beam include the permanent CMOD or central displacement set values, as obtained from the first loading cycle. Both of these methods of plotting the data are illustrated in Figure 10. This figure shows further evidence of the primary healing effect, both by the increased stiffness response of the SH beam following tube breakage, and the increased stiffness of this beam during unloading. The latter results in an approximate doubling of the permanent set value for the SH beam, compared with the control beam, following unloading $(0.29 \mathrm{~mm}$ compared to $0.15 \mathrm{~mm})$. The reason for the stiffer unloading response is believed to be due to the rapid curing and hardening of the adhesive that has entered the crack, which then serves to act as a 'wedge' between the crack surfaces on unloading.

Interestingly, on reloading after a period of $24 \mathrm{~h}$, the high level of stiffness observed during unloading of the SH specimen is maintained, as illustrated by line ' $d$ ' in Figure 10(b). If the gradient of SH beam response ' $d$ ' is compared with the gradient of the control response ' $\mathrm{e}$ ', then it is clear that the $0.3 \mathrm{~mm}$ crack created during the first loading cycle has been healed. The reloading of the SH specimen must, therefore, result in new crack formation (see Figure 15 later), or a peeling apart of the newly bonded original crack faces. As a result of the above, the hysteresis loop for the SH beam is also therefore far less than that of the control beam. 


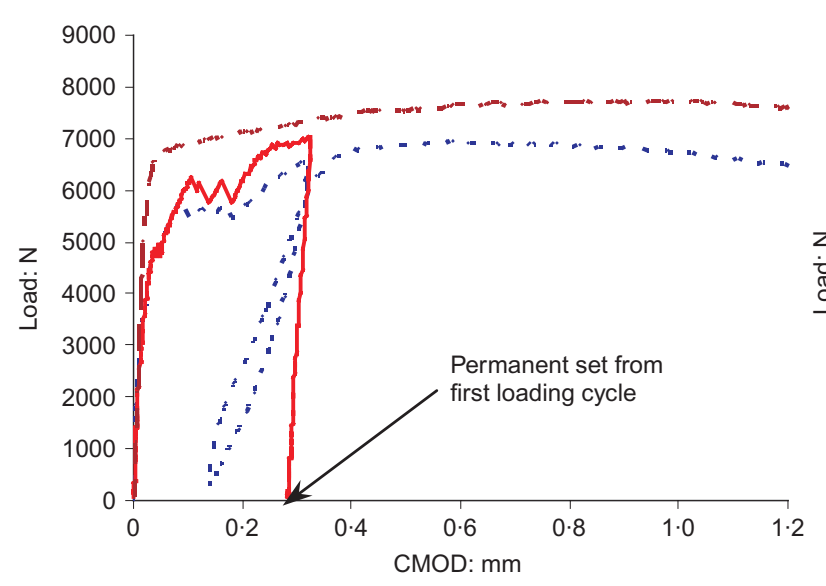

(a)

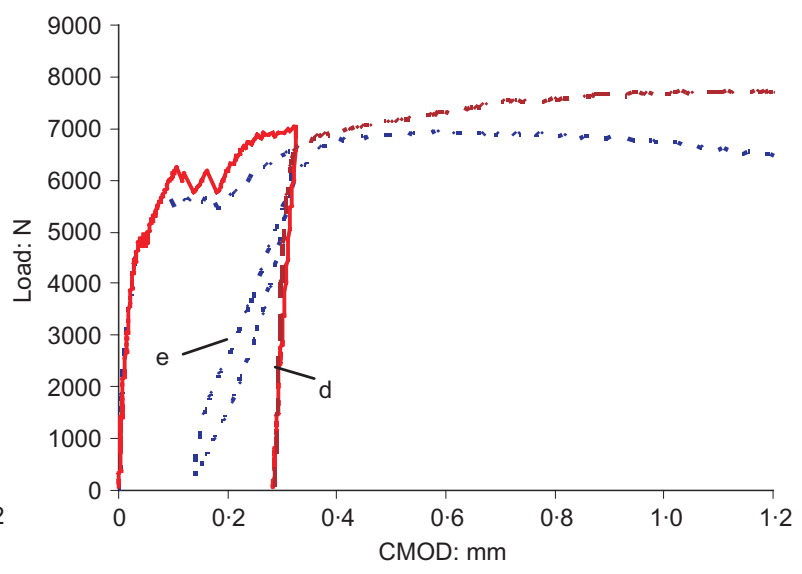

(b)
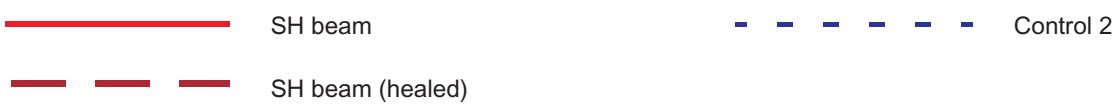

Figure 10. Load-CMOD response for SH beam 1 and control beam 2 from set 2 (a) without and (b) with the permanent set from the first loading cycle considered

In Figure 10(a) the permanent set obtained from the first loading cycle on the $\mathrm{SH}$ beam is removed, and the second loading cycle response is plotted from the origin. The response of this set $2 \mathrm{SH}$ beam post-healing, when compared with the virgin response of the control beam, is similar to that illustrated previously for the SH beam from set 1 (Figure 9(a)), and once again shows an increase in stiffness, peak strength and postpeak ductility. This apparent 'enhancement' of the post-healed beam properties, when compared with the virgin properties, is believed to be attributable to two reasons.

(a) The very low viscosity cyanoacrylate is believed to not only flow into the crack and towards the crack tip under the influence of capillary forces but is also believed to infiltrate the region of micro-cracks within the FPZ (fracture process zone), behind the crack faces, thus creating a cementitious-polymer composite, with improved mechanical properties, in the central region of the beam.

(b) Since the loading arrangement employed in the current experimental series creates a peak bending moment at the specimen centre-span, then the final macro crack created during the second loading cycle is forced to propagate through this mechanically improved zone, or deviate significantly around it.

\section{Effect of reinforcement level}

Three levels of beam reinforcement have been examined in the experimental programme, as detailed in Table 3. These include reinforcement configurations consisting of a single $3.15 \mathrm{~mm}$ bar, two $3.15 \mathrm{~mm}$ bars, and a single $6.7 \mathrm{~mm}$ diameter bar. The levels of rein- forcement provided by each of these configurations are $0 \cdot 16 \%, 0 \cdot 32 \%$ and $0 \cdot 72 \%$, respectively.

The force-CMOD response for typical SH beams from sets 2, 3 and 4 are given in Figure 11. The strength of the control beams, after initial cracking of the mortar and glass tubes, increases with an increase in the percentage of reinforcement, as expected.

The main effect that the level of reinforcement has on the self-healing behaviour is evident in the gradient of the primary healing response that occurs immediately after the fracture of the glass tubes. Figure 11 shows that as the level of reinforcement increases so does the gradient of the primary healing line. This is believed to be attributable to the rate at which the macro crack opens. For higher levels of reinforcement the rate of crack opening is slower, and therefore, the adhesive, which has flowed into the crack, and is in the process of hardening, is effectively being loaded at a slower rate. When an adhesive layer is being loaded in tension at the same time as it is curing, a slower loading rate will result in a stiffer response, since the bonds have more time to form.

\section{Effect of loading rate}

Three different rates of loading have been examined in set 5 , each of which are four times faster than the previous loading rate. The rates used for both the first and second loading cycles are $0.00075 \mathrm{~mm} / \mathrm{s}$, $0.003 \mathrm{~mm} / \mathrm{s}$ and $0.012 \mathrm{~mm} / \mathrm{s}$. The force-CMOD response for three typical $\mathrm{SH}$ beams from set 5 are given in Figure 12.

The main effect of the loading rate is also evident in the gradient of the primary healing response; as the monotonic rate of stroke-controlled loading is increased, the stiffness of the primary healing response 


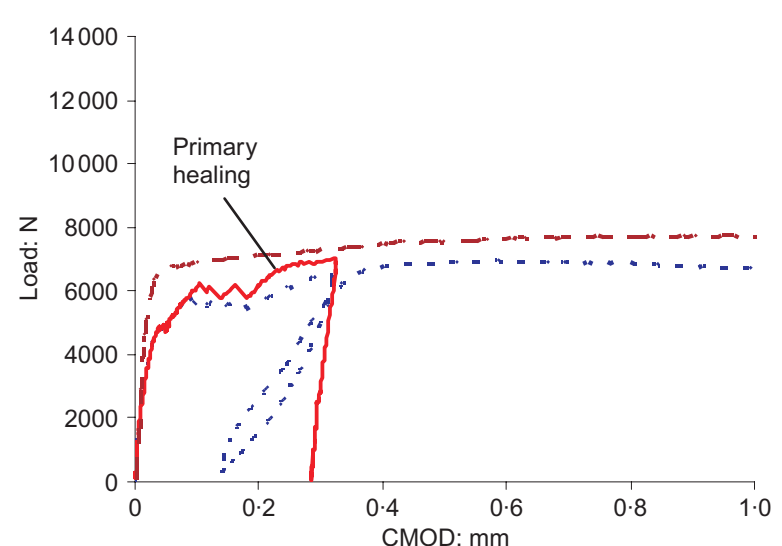

(a)

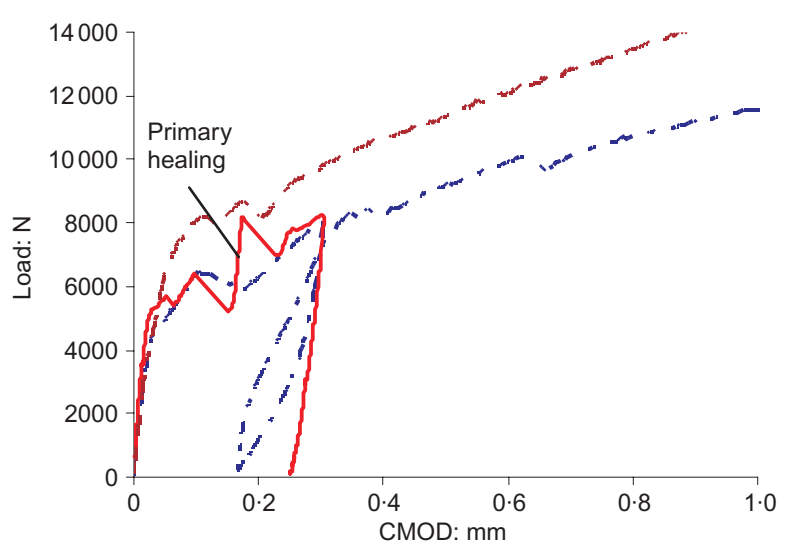

(c)

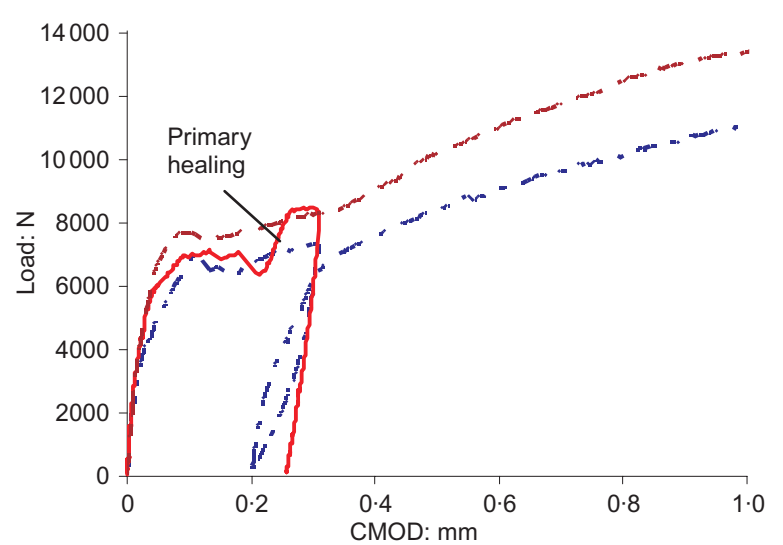

(b)

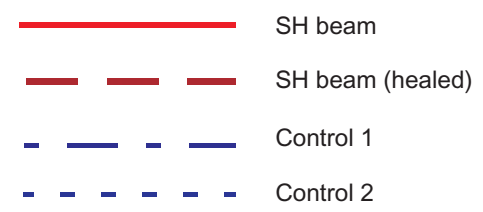

Figure 11. Load-CMOD response for (a) SH beam 1 from set 2, with a single $3.15 \mathrm{~mm}$ reinforcing bar, (b) SH beam 3 from set 3, with two $3.15 \mathrm{~mm}$ reinforcing bars and (c) SH beam 4 from set 4, with a single $6.7 \mathrm{~mm}$ reinforcing bar

decreases. This trend is again believed to be attributable to the effect that the loading rate has on the stiffness response of the layer of adhesive which is hardening between the crack faces, as outlined in the previous section. This effect is absent in the response of the control specimens.

\section{Effect of specimen age}

As noted in Table 3, sets 1-3 have been tested at 28 days, while sets 4 and 5 have been tested at an age of 70 days. SH beams 4, 5 and 6 from experimental set 5 may therefore be compared with the $\mathrm{SH}$ beams from set 2 to ascertain whether the age of the specimen has a notable effect on its autonomic healing ability. It may be concluded, from comparison of these experimental results, that there is no indication of the specimen age affecting the self-healing response. This is not unsurprising since, unlike autogenous healing, which is intrinsically linked to the age of the specimen (Schlangen et al., 2006), the autonomic healing response is dependent primarily on the viscosity and curing abilities of the adhesive and the suitability of the delivery system.

\section{Qualitative results}

Ink and glue flow. During all first cycle tests the 840 distinct fracturing sound of the four capillary tubes was heard. For the control beams this was followed within $20-30 \mathrm{~s}$ by ink staining on the side faces of the beams (Figure 13(a)), indicating the presence of the capillary suction effect caused by the propagating crack.

The flow of cyanoacrylate during loading of the selfhealing specimens was less pronounced than the ink tracing die, owing to its colourless appearance and higher viscosity; however, Figure 13(b) clearly indicates that the adhesive has flowed from the capillary tubes and has entered the crack. It should be noted that despite there being a large amount of adhesive available within the reservoir (approximately $10 \mathrm{ml}$ ), only about $0 \cdot 1-0.3 \mathrm{ml}$ was initially observed to flow into the crack, following the fracture of the capillary tubes. This value was estimated from the observed glue level drop in the curved supply tubes shown in Figure 6. A further drop in the glue level was then generally recorded as the crack widened and surplus adhesive was observed to flow from the underside of the beam in the locality of the notch (Figure 13(b)).

It should also be noted that despite the rapid curing abilities of cyanoacrylate, when deposited in thin layers which are in contact with moisture and oxygen, when 


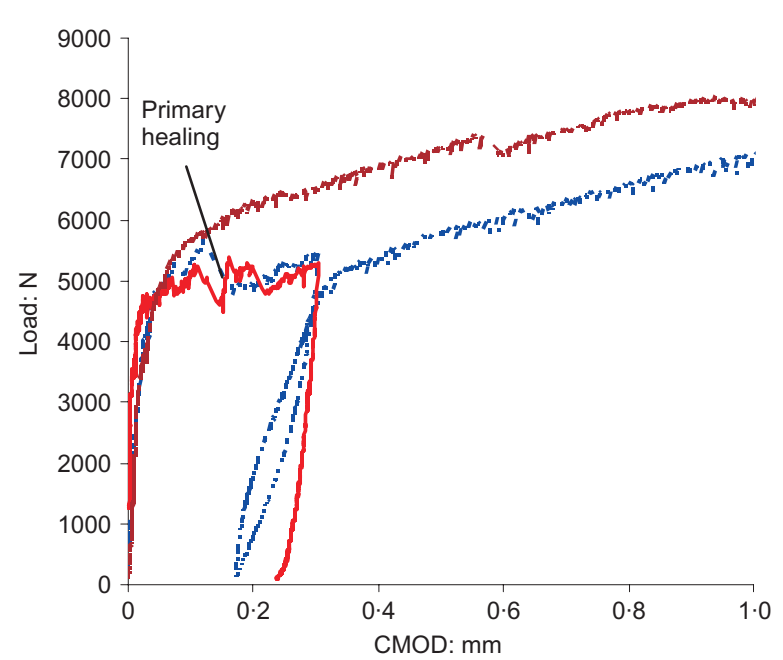

(a)

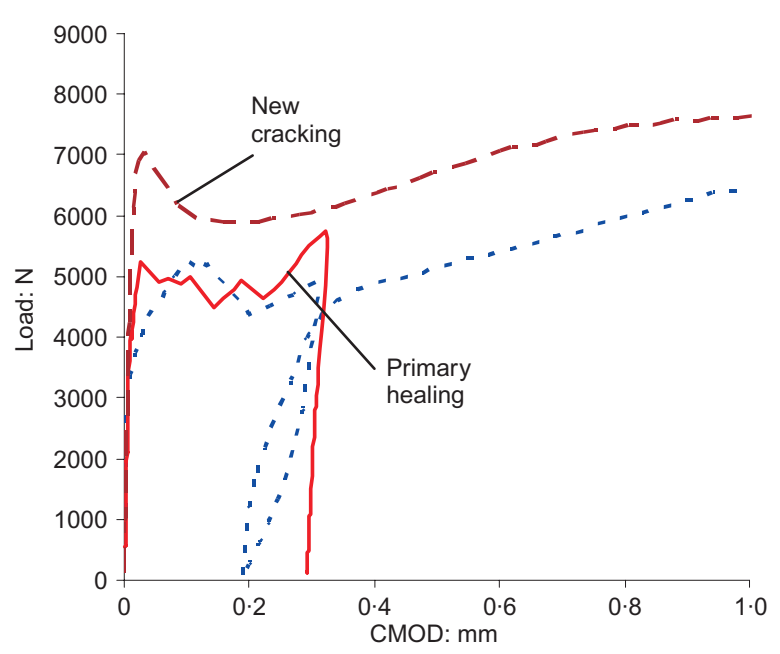

(c)

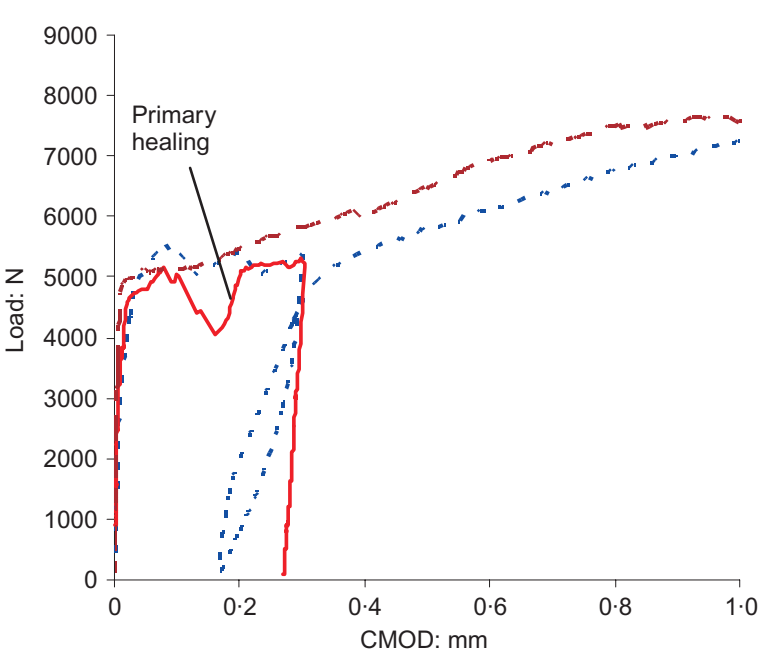

(b)

Figure 12. Load-CMOD response for (a) SH beam 1 from set 5, tested at a rate of $0.00075 \mathrm{~mm} / \mathrm{s}$, (b) SH beam 4 from set 5 , tested at a rate of $0.003 \mathrm{~mm} / \mathrm{s}$ and (c) SH beam 7 from set 5 , tested at a rate of $0.012 \mathrm{~mm} / \mathrm{s}$

stored in larger volumes its curing rate is significantly slower. The cyanoacrylate which remained in the supply tubes following testing was observed to remain in liquid form for over a week, despite being open to the atmosphere. As a result, many of the specimens released additional adhesive during the second loading cycle. It may therefore be possible to capture a tertiary healing effect from the fixed supply system used in these experiments, although this has not been investigated in the current work.

Confirmation of glue flow into the crack is given by the staining patterns shown in Figure 14. Similar glue migration patterns were obtained for all of the selfhealing beams tested. Figure 14 indicates that the extent of the spread of the adhesive is linked to the effectiveness of the mechanical healing results obtained from the three-point bend tests. SH beam 1 exhibited very little self-healing owing to specific problems with its supply system, and as a result the adhesive capillary rise appears to have been in the order of only $10 \mathrm{~mm}$. Conversely, SH beam 4, which exhibited increased stiffness, peak strength and ductility (Figure 9) shows a significantly greater adhesive capillary rise of approximately $30 \mathrm{~mm}$.

New crack formation. Further qualitative evidence of the self-healing process is also given by new crack formation which has been observed to occur during the second loading cycle, as shown in Figure 15, see later.

Despite the stress concentration created by the notch, the final cracking path for the self-healing specimens is different from the original macro-crack created during the first loading cycle. This new crack formation, which was not observed to occur in either of the control specimens, demonstrates the bonding capabilities of the ethyl cyanoacrylate when used within a mortar matrix. 


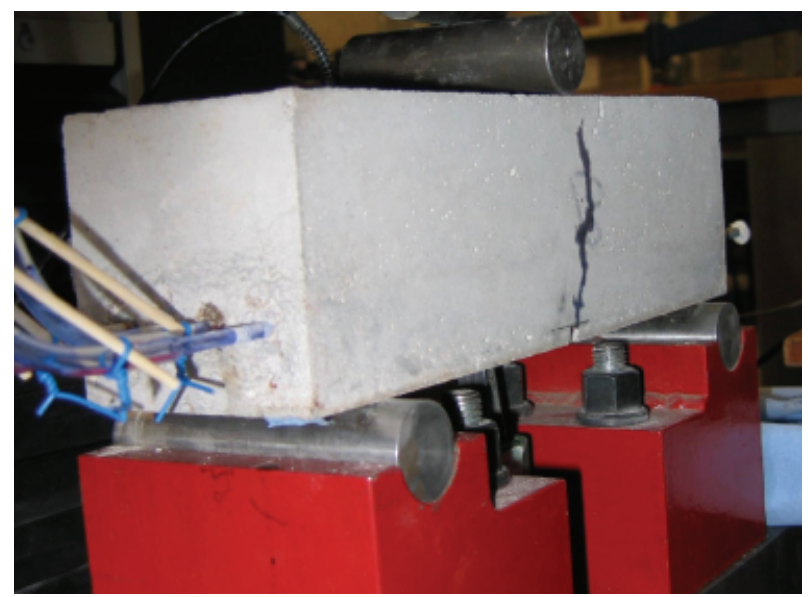

(a)

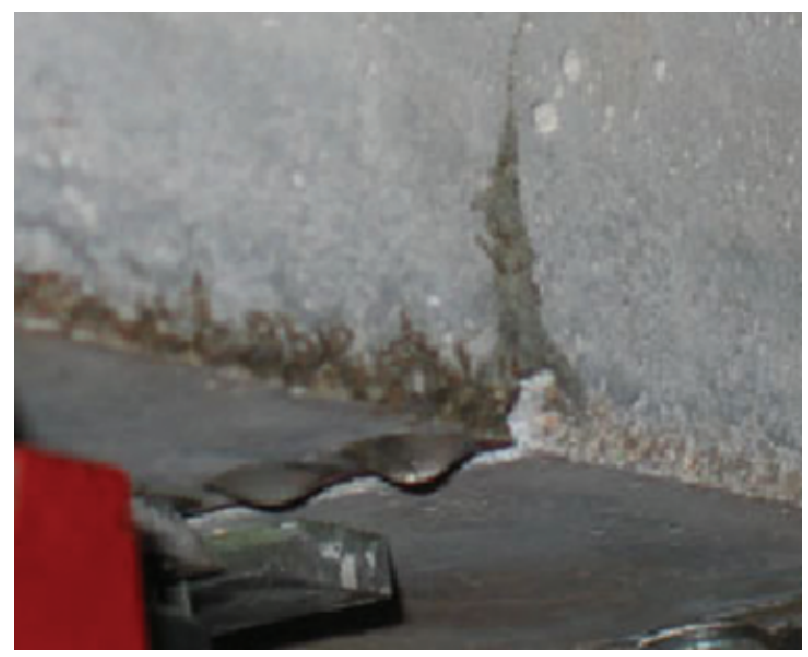

(b)

Figure 13. (a) Ink migration during testing of lightly reinforced, notched control specimen; (b) glue flow during testing of a lightly reinforced, notched self-healing specimen

\section{Conclusions}

The main conclusions of the preliminary self-healing investigation work presented in this paper were that

(a) a low-viscosity, single-agent, cyanoacrylate adhesive offered the greatest potential in a healing system which relies on capillary flow

(b) a fully encapsulated delivery system (adhesive sealed within short lengths of hollow capillary tubes) did not allow the adhesive to flow out following cracking owing to the negative pressure force generated by the end wax plugs.

The final five sets of experiments therefore utilised a delivery system incorporating capillary tubes which were open to the atmosphere. These tests served to investigate the nature of the adhesive-based healing response within mortar beams, and the effect of the reinforcement level and loading rate on this self-healing response.

Evidence has been given of both a primary and 842

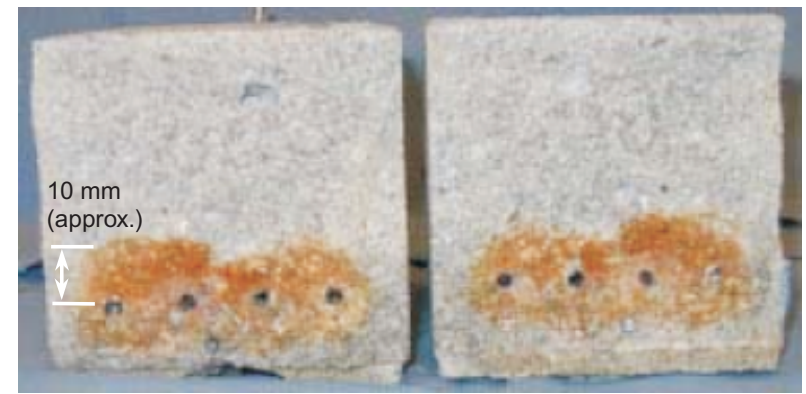

(a)

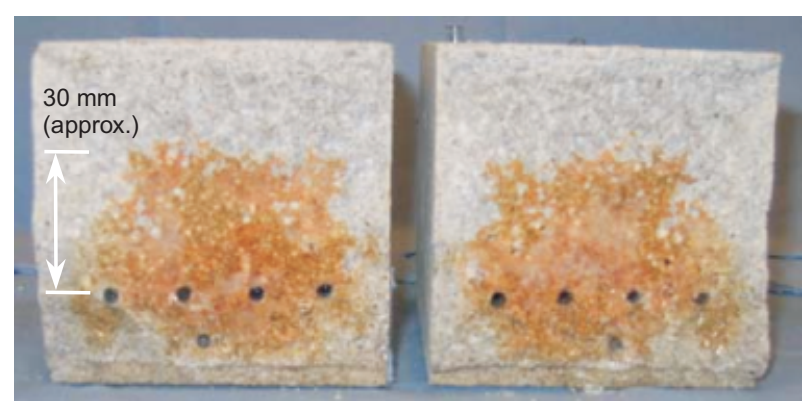

(b)

Figure 14. Glue migration and effective zone of healing for experimental set 1 on notched, lightly reinforced mortar beams: (a) SH beam 1 and (b) SH beam 4
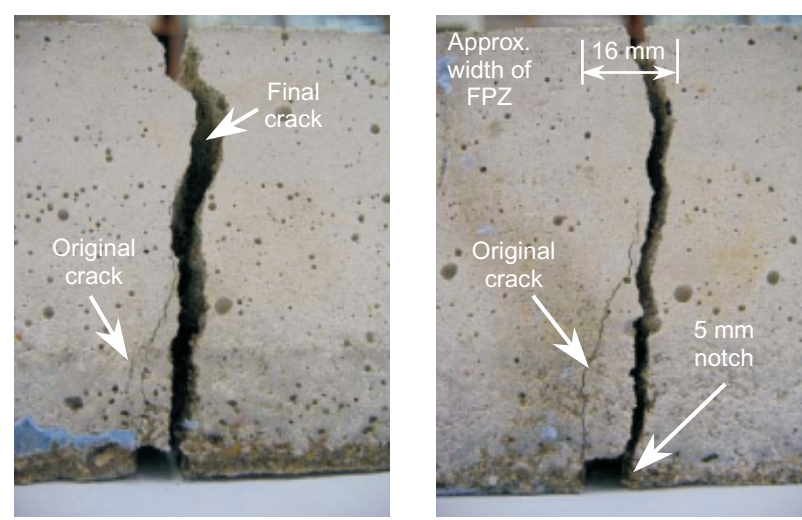

Figure 15. Original and final crack patterns on the side face of (a) SH beam 2 and (b) SH beam 3, for the first

experimental round on notched, lightly reinforced beams

secondary healing response, occurring during the first and second loading cycles, respectively. In addition, the stiffness of the primary healing response has also been found to increase with increasing reinforcement percentage and decreasing loading rate.

Qualitative evidence of the efficacy of the self-healing system has also been obtained, including: distinct cracking of the capillary tubes followed by immediate release of the adhesive from the supply reservoir; glue migration patterns on the cracked faces, and new crack formation during the second loading cycle.

Future publications will describe work on the adhesive-based self-healing system for unnotched

Magazine of Concrete Research, 2010, 62, No. 11 
beams, and on the development of a numerical model to capture the self-healing process. In addition, future development work is also required on the adhesive delivery system in order to improve its practicality for reinforced concrete structures. It is envisaged that such systems would involve a network of tubes, possibly attached to the reinforcement, through which adhesive would be circulated under pressure.

\section{Acknowledgements}

The authors would like to acknowledge: Professor Bhushan Karihaloo for introducing them to the area of self-healing cementitious materials; Mauro Cantoni for his assistance with the preliminary investigation work; and the laboratory personnel at the School of Engineering for their technical support.

\section{References}

3M (2007) Rite-Lok cyanoacrylate adhesive EC5 product data sheet. 3M Ltd, Maplewood, MN, USA.

BSI (British Standards Institution) (2005) BS 4449: Steel for the reinforcement of concrete. Weldable reinforcing steel. Bar, coil and decoiled product. Specification. BSI, London.

CEN (Comité Européen de Normalisation) (2004) BS EN 1992. Eurocode 2: Design of concrete structures - Part 1-1: General rules and rules for buildings. CEN, Brussels.

Dry C (1994) Matrix cracking repair and filling using active and passive modes for smart timed release of chemicals from fibres into cement matrices. Smart Materials and Structures 3(2): 118123.

Dry C (2006) Self-Repairing, Reinforced Matrix Materials. US Patent 7022179 .
DTI (Department for Trade and Industry) (2006) Construction Statistics Annual Report 2006. TSO, London.

Joseph C (2008) Experimental and Numerical Study of the Fracture and Self-healing of Cementitious Materials. PhD thesis, Cardiff University.

Joseph C, Jefferson AD and Cantoni MB (2007) Issues relating to the autonomic healing of cementitious materials. Proceedings of the 1st International Conference on Self-Healing Materials, Noordwijk, the Netherlands. CD-ROM.

Kepler WF, Von F and Kurt F (2001) Micro-encapsulated Crack Resistant Cement. US Patent 6565644.

Li VC, Lim YM and Chan YW (1998) Feasibility study of a passive smart self-healing cementitious composite. Composites Part B: Engineering 29(B): 819-827.

Mihashi H, Kaneko Y, Nishiwaki T and Otsuka K (2000) Fundamental study on development of intelligent concrete characterized by self-healing capability for strength. Transactions of the Japan Concrete Institute 22(2): 441-450.

Nishiwaki T, Mihashi H, Jang B-K and Miura K (2006) Development of self-healing system for concrete with selective heating around crack. Journal of Advanced Concrete Technology 4(2): 267-275.

Schlangen E, Ter Heide N and van Breugel K (2006) Crack healing of early age cracks in concrete. In Measuring, Monitoring and Modelling Concrete Properties. Springer, the Netherlands, pp. $273-284$.

van der Zwaag S (2007) Self-healing Materials: An Alternative Approach to 20 Centuries of Material Science. Springer, the Netherlands.

van Oss HG (2005) Background Facts and Issues Concerning Cement and Cement Data. US Department of the Interior and US Geological Survey. Open-file report 2005-1152.

White SR, Sottos NR, Geubelle PH et al. (2001) Autonomic healing of polymer composites, Nature 409: 794-797.

Williams G, Trask R and Bond I (2007) A self-healing carbon fibre reinforced polymer for aerospace applications. Composites: Part A 38(6): $1525-1532$.

Discussion contributions on this paper should reach the editor by 1 May 2011 